\title{
Anlotinib vs. Nanoparticle Albumin-Boundpaclitaxel in the Treatment of Third-Line and Above Extensive Stage Small Cell Lung Cancer
}

\section{Li Li}

Nanjing Brain Hospital

Qiaohong Bai

Nanjing Second Hospital

\section{Yuchao Wang}

Nanjing Brain Hospital

Wei Liu

Nanjing Brain Hospital

Chunhua Xu ( $\nabla$ xuch2188@163.com )

Nanjing Brain Hospital

Chuanzhen Chi

Nanjing Brain Hospital

\section{Research Article}

Keywords: Anlotinib, Nanoparticle albumin-boundpaclitaxel, third-line therapy, small cell lung cancer, Adverse reactions

Posted Date: September 3rd, 2021

DOl: https://doi.org/10.21203/rs.3.rs-785658/v1

License: (9) This work is licensed under a Creative Commons Attribution 4.0 International License. Read Full License 


\title{
Anlotinib vs. Nanoparticle albumin-boundpaclitaxel in the treatment of third-line and above extensive stage small cell lung cancer
}

Li $\mathrm{Li}^{1}$, 2, 3, Qiaohong $\mathrm{Bai}^{4}$, Yuchao Wang ${ }^{1,2,3}$, Wei $\mathrm{Liu}^{1,2,3}$,Chunhua $\mathrm{Xu}^{1}{ }^{1,2,3}$, Chuanzhen $\mathrm{Chi}^{1,2,3}$

${ }^{1}$ Department of Respiratory Medicine, Nanjing Chest Hospital, Nanjing, Jiangsu 210029, China

${ }^{2}$ Affiliated Nanjing Brain Hospital, Nanjing Medical University, Nanjing, Jiangsu 210029, China

${ }^{3}$ Clinical Center of Nanjing Respiratory Diseases and Imaging, Nanjing, Jiangsu 210029, China

${ }^{4}$ Department of Respiratory Medicine, The Second Hospital of Nanjing,Nanjing University of Chinese Medicine, Nanjing 210003, China

\section{Correspondence}

Chunhua $\mathrm{Xu}$, Department of Respiratory Medicine, Nanjing Chest Hospital, 215 Guangzhou Road, Nanjing 210029, China.

E-mail: xuch2188@163.com.

Chuanzhen Chi, Department of Respiratory Medicine, Nanjing Chest Hospital, 215 Guangzhou Road, Nanjing 210029, China.

E-mail: chuanzhenchi@163.com.

\begin{abstract}
Background: Comparison of the efficacy and adverse reactions of anlotinib and nanoparticle albumin-boundpaclitaxel (Nab-P) in the treatment of third-line and above extensive-stage small cell lung cancer (ES-SCLC).
\end{abstract}

Methods: In this study, we retrospectively analyzed the effectiveness and safety of 
anlotinib and Nab-P in clinical practice. We enrolled 71 patients with ES-SCLC who received anlotinib monotherapy or Nab-P monotherapy as third-line or later-line therapy. Divided into Anlotinib group $(n=35)$ and Nab-P group $(n=36)$. We analyzed progression-free survival (PFS), overall survival (OS), objective response rate (ORR), disease control rate (DCR), and adverse events (AE) in the two groups.

Results: Among all patients, the Nab-P group showed better overall response rate (ORR) compared with the Anlotinib group [19.4\% vs. 8.6\%, $P=0.193]$. There was no significant difference in PFS and OS between the two groups. The most common AEs in the Anlotinib group were liver damage, fatigue, hypertension, and hand-foot syndrome. The most common AEs in the Nab-P group were liver damage, neutropenia, fatigue, and muscle aches. There were no treatment-related deaths.

Conclusions: In our real-world data analysis, patients treated with Nab-P had a better overall response rate (ORR), but no benefit of PFS or OS. Both treatment options are effective, so the choice should be based on their potential side effects.

Keywords: Anlotinib, Nanoparticle albumin-boundpaclitaxel, third-line therapy, small cell lung cancer, Adverse reactions

\section{Background}

Small cell lung cancer (SCLC) accounts for about $15 \%$ of all pathological types of lung cancer. It is characterized by high malignancy, easy recurrence and metastasis, and sensitivity to chemotherapy and radiotherapy. Compared with non-small cell lung cancer (NSCLC), its median survival time is only $8-10$ months, and more than $95 \%$ of patients die within 2 years $[1,2]$. Chemotherapy is still the cornerstone of the 
treatment of extensive-stage small cell lung cancer. Although the guidelines have recommended immunotherapy combined with chemotherapy as the first-line treatment for extensive-stage small cell lung cancer, extensive-stage small cell lung cancer still has to face second-line, third-line and even multiple lines after recurrence and metastasis treatment. At present, the third-line and multi-line treatment of small cell lung cancer is not clear. Studies had shown that Anlotinib is effective in the third-line treatment of small cell lung cancer [3, 4], and studies had also shown that Nab-P had a certain effect in the treatment of small cell lung cancer $[5,6]$. In order to provide the best option for the treatment of third-line and above ES-SCLC, we studied the efficacy of anlotinib single agent versus Nab-P in the real world in patients with ES-SCLC.

\section{Methods}

We retrospectively collected 71 patients with ES-SCLC who were admitted to the Department of Respiratory Medicine of our hospital from January 2016 to December 2020. The inclusion criteria were as follows: 1) The imaging and pathological diagnosis and staging of ES-SCLC; 2) Patients who had undergone at least second-line chemotherapy in the past; 3) Patients who had at least one evaluable lesion; 4) Follow-up was continuous.

The patients enrolled were divided into two groups, namely Anlotinib group and Nab-P group. The Anlotinib group was treated with anlotinib $12 \mathrm{mg}$ per day as a single agent for 14 consecutive days and stopped for 7 days. 21 days was a cycle, and at least two cycles of targeted therapy were completed; Nab-P group was given 
single-agent chemotherapy of Nab-P, and the dose was $130 \mathrm{mg} / \mathrm{m}^{2}$ intravenously on the first and eighth days. 21 days was one cycle, the patient had completed at least two cycles of chemotherapy.

This study was approved by the Association of Thoracic Ethics Clerks in the Affiliated Brain Hospital of Nanjing Medical University. All aspects of the research were consistent with the guidelines of the Declaration of Helsinki. All patients in this study participate voluntarily, and all patient data were strictly confidential. As it was a retrospective study, it was specifically approved by the Thoracic Ethics Committee of the Brain Hospital Affiliated to Nanjing Medical University without written consent.

\section{Data Collection}

We collected the basic clinical information of the enrolled patients through the hospital's electronic medical record system, including age, gender, ECOGPS score, previous treatment status, and efficacy evaluation. We followed up by phone. The efficacy evaluation refered to International Solid Tumor (RECIST) 1.1, which was divided into complete remission (CR), partial remission (PR), stable disease (SD), and disease progression (PD). $\mathrm{CR}+\mathrm{PR} /$ total number of cases was calculated the objective response rate $(\mathrm{ORR}), \mathrm{CR}+\mathrm{PR}+\mathrm{SD} /$ total number of cases was the disease control rate (DCR). PFS was defined as the time from the start of treatment to the first confirmation of disease progression, while OS was defined as the time from the start of treatment to death. According to the common toxicity standard version 4.0 of adverse events, toxic and side effects were classified.

\section{Statistical Analysis}


Apply SPSS19.0 and StataSE12.0 to analyze all data. The basic information of the two groups was compared by chi-square test, the curative effect was compared by Kaplan-Meier method and log-rank test. Forest plots were used for subgroup analysis. Set the statistical significance to $\mathrm{P}<0.05$.

\section{Results}

\section{Clinicopathologic Characteristics}

Between January 2016 and December 2020, a total of 71 patients with extensive-stage small cell lung cancer who had undergone at least second-line chemotherapy.This was a retrospective real-world study comparing anlotinib single agent (35 cases in the Anlotinib group) and Nab-P single agent (36 cases in the Nab-P group) in the treatment of third-line and above ES-SCLC. The median age was 63 (45-82) years in the Anlotinib group and 62 (33-78) years in the Nab-P group. Anlotinib and Nab-P groups enrolled evenly distributed of male, immunotherapy, elder, ever smoking, and metastases in various organs $(\mathrm{P}>0.05)$. In terms of previous treatment, the proportion of patients with fourth-line and above in the Anlotinib group was larger than Nab-P group (68.6 vs. 30.6, $\mathrm{P}<0.05)$ (Tabel 1).

\section{Treatment and Efficacy}

Patients in the anlotinib group received $12 \mathrm{mg}$ anlotinib monotherapy daily. If serious side effects occur, reduce the dose to $10 \mathrm{mg}$ per day or even stop the drug. The Nab-P group was given single-agent chemotherapy of Nab-P with a dose of $130 \mathrm{mg} / \mathrm{m}^{2}$ intravenously on the first and eighth days. After 2 cycles, the short-term efficacy was evaluated according to the RECIST standard. For patients were without CR in both 
groups, DCR was also similar. The ORR of the Nab-P group was higher than that of the Anlotinib group (19.4\% vs. $8.6 \%, P=0.193$ ) (Table 2). There was no significant difference between the Anlotinib group and the Nab-P group in PFS and OS. (median PFS, 5.0 vs. 5.0 months; HR [95\% CI]: 0.377 [4.26 - 5.74], $P=0.664$; median OS, 9.4 vs. 9.2 months; HR [95\% CI]: 0.996 [7.45 - 11.35], $P=0.627$ ) (Fig. 1).

\section{Subgroup Analysis}

In order to confirm the influence of each clinical feature on the PFS and OS of the two groups, we conducted a subgroup analysis. The subgroup analysis of the two groups for PFS suggested that patients with ECOGPS (0-1) could obtain longer PFS in the Nab-P group $(\mathrm{P}<0.05)$. There was no significant difference between the two groups in subgroup analysis of OS $(\mathrm{P}>0.05)$ (Figure 2).

\section{Adverse Events}

There were some differences in the incidence of adverse events between the two groups. The incidence of hypertension, Thrombosis and hemorrhage, and hand-foot syndrome in the Anlotinib group were significantly higher than that in the Nab-P group $(P<0.05)$. The incidence of neutropenia, peripheral neuropathy, and thrombocytopenia in the Nab-P group were all Higher than the Anlotinib group. Among them, the comparison of neutropenia between the two groups had important statistical differences $(P<0.05)$. The highest incidence of adverse events in the two groups was abnormal liver function. The overall incidence of adverse events in the two groups, including those of grade $\geqslant 3$, were similar, and there was no difference. The safety of the two groups of drugs was tolerable, and there were no drug-related 
deaths (Table 3).

\section{Discussion}

Small cell lung cancer is a highly malignant tumor that is prone to recurrence and metastasis. Chemotherapy is still the main weapon in the treatment of small cell lung cancer. Most patients with small cell lung cancer will relapse soon after the initial treatment and develop drug resistance. Although the remission rate after the first chemotherapy can reach more than $80 \%$, the median PFS and OS are short, and the prognosis is poor [7]. The current guidelines have clear recommendations for the first-line and second-line treatment of ES-SCLC, but the treatment of third-line and above is not clear. Anlotinib is a multi-target inhibitor, including Vascular endothelial growth factor receptor (VEGFR), platelet-derived growth factor receptor (PDGFR), fibroblast growth factor receptor (FGFRR) and tyrosine kinase (c-Kit) pathway [8]. In the ALTER 1202 study, anlotinib was treated with third-line and above for ES-SCLC, and obtained 4.1 months of PFS and 7.3 months of OS [9]. The anti-tumor mechanism of paclitaxel was to block the $\mathrm{M}$ phase of the cell cycle by inhibiting the depolymerization of tubulin, thereby blocking cell division. Nab-P transports more paclitaxel to the tumor site through its unique albumin, which can achieve high efficiency and low toxicity $[10,11]$. Studies had shown that the ORR of Nab-P monotherapy in patients with second-line drug-resistant small cell lung cancer was $33 \%$ [12]. In order to choose the best treatment plan in the third-line and above treatments of ES-SCLC in clinical practice, we compared anlotinib and Nab-P single-agent treatments in the real world for the treatment of patients with ES-SCLC. 
The Nab-P group improved ORR (19.4\% vs. $8.6 \%$ ), although there was no statistical difference in PFS and OS between the two groups. In our aanlotinib group, the median PFS was 5 months and the median OS was 9.4 months. The median PFS in the Nab-P group was 5 months, and the median OS was 9.6 months. This result was better than previous studies of anlotinib ALTER 1202 and second-line treatment of SCLC with Nab-P. The clinical characteristics of the two groups of patients were basically the same. The fourth-line and later patients in the Anlotinib group accounted for a higher proportion, while the third-line patients in the Nab-P group accounted for a higher proportion. In the subgroup analysis, in terms of PFS, patients with an ECOGPS score of $0-1$ were more benefited in the Nab-P group $(P<0.05)$. In the subgroup analysis of OS, there was no significant difference between the two groups. The overall incidence of adverse events in the two groups was similar, but there were also certain differences. The incidence of hypertension, thrombosis and hemorrhage, and hand-foot syndrome was significantly higher in the Anlotinib group, and the incidence of neutropenia, peripheral neuropathy, and thrombocytopenia was higher in the Nab-P group $(P<0.05)$. This was similar to the adverse reaction results of previous studies [13-16].

In the past, there were no similar studys on the treatment of ES-SCLC with anlotinib versus Nab-P. In clinical work, we should make comprehensive assessment and individualized treatment based on the patient's specific conditions and the side effects of the two drugs. Choose the most suitable treatment plan for each patient.

At present, immunotherapy has brought hope for the treatment of small cell lung 
cancer. Follow-up combined immunotherapy with anlotinib or Nab-P in the future treatment of ES-SCLC is also worth looking forward to.

Our study has certain limitations. It was a single-center retrospective study, and there were differences between patients. I look forward to more real-world studies in the future to provide more basis for the choice of clinical treatment.

\section{Conclusion}

In the third-line and above treatments of ES-SCLC, both anlotinib and Nab-P monotherapy are effective and tolerable. The ORR of Nab-P was higher, and patients with an ECOGPS score of 0-1 could choose Nab-P to obtain longer PFS. Clinically, suitable drugs are selected according to the condition of the disease and the side effects of the drugs.

\section{Abbreviations}

Nab-P: nanoparticle albumin-boundpaclitaxel; ES-SCLC: extensive-stage small cell lung cancer; ECOG PS: eastern cooperative oncology group performance status; CR: complete response; PR: partial response; SD: stable disease; PD: progressive disease; PFS: progression-free survival; OS: overall survival; DCR: disease control rate; ORR: objective Response Rate.

\section{Acknowledgements}

Not applicable.

\section{Funding}

The current study was supported by the Major Program of Nanjing Medical Science and Technique Development Foundation (ZKX17044).

\section{Availability of data and materials}


All data are available by contacting correspondence.

authors: via: xuch2188@163.com

\section{Authors' contributions}

CHX, LL, CZC and YCW contributed to the study concept and design. LL performed the ultrasound exams and drafted the article. QHB and YCW contributed to the analysis and interpretation of the data. CHX and WL take responsibility for the integrity of this work as a whole. All authors read, commented on, and contributed to the submitted manuscript. All authors read and approved the final manuscript.

\section{Ethics approval and consent to participate}

This study was approved by the Ethics Clerk Association of the Thoracic Department of the Brain Hospital Affiliated to Nanjing Medical University. All aspects of the study are in line with the guidelines of the Declaration of Helsinki. In this study, patient participated voluntarily, and all patient data were strictly confidential. Because it is a retrospective study, the Ethics Committee of the Thoracic Department of the Brain Hospital Affiliated to Nanjing Medical University has specifically approved it without written consent.

\section{Consent for publication}

Not applicable.

\section{Competing interests}

The authors declare that they have no competing interests.

\section{References}

1. Kalemkerian GP, Schneider BJ. Advances in Small Cell Lung Cancer. Hematol 
Oncol Clin North Am. 2017;31:143-156.

2. Koinis F, Kotsakis A, Georgoulias V. Small cell lung cancer (SCLC): no treatment advances in recent years. Transl Lung Cancer Res. 2016;5:39-50.

3. Chen D, Xu J, Zhao Y, Chu T, Zhong H, Han B, et al. Prognostic value of tumor cavitation in extensive-stage small-cell lung cancer patients treated with anlotinib. $\mathrm{J}$ Cancer Res Clin Oncol. 2020;146:401-406.

4. Song PF, Xu N, Li Q. Efficacy and Safety of Anlotinib for Elderly Patients with Previously Treated Extensive-Stage SCLC and the Prognostic Significance of Common Adverse Reactions. Cancer Manag Res. 2020;12:11133-11143.

5. Nakao M, Fujita K, Suzuki Y, Arakawa S, Sakai Y, Sato H, et al. Nab-paclitaxel Monotherapy for Relapsed Small Cell Lung Cancer: Retrospective Analysis and Review. Anticancer Res. 2020;40:1579-1585.

6. Yamamoto N, Tsurutani J, Yoshimura N, Asai G, Moriyama A, Nakagawa K, et al. Phase II study of weekly paclitaxel for relapsed and refractory small cell lung cancer. Anticancer Res. 2006;26:777-81.

7. Spigel DR. Treatment update in small-cell lung cancer: from limited to extensive disease. Curr Treat Options Oncol. 2012 Dec;13(4):505-15.

8. Xie C, Wan X, Quan H, Zheng M, Fu L, Li Y, et al. Preclinical characterization of anlotinib, a highly potent and selective vascular endothelial growth factor receptor-2 inhibitor. Cancer Sci. 2018;109:1207-1219.

9. Zhang C, Wang J, Wang X, Meng Z, Cheng Y, Li K. Peripheral blood indices to predict PFS/OS with anlotinib as a subsequent treatment in advanced small-cell lung 
cancer. Cancer Biol Med. 2021:j.issn;2095-3941.

10. Kundranda MN, Niu J. Albumin-bound paclitaxel in solid tumors: clinical development and future directions. Drug Des Devel Ther. 2015;9:3767-77.

11. Desai N, Trieu V, Yao Z, Louie L, Ci S, Yang A, et al. Increased antitumor activity, intratumor paclitaxel concentrations and endothelial cell transport of cremophor-free, albumin-bound paclitaxel, ABI-007, compared with cremophor-based paclitaxel. Clin Cancer Res. 2006;15:1317-1324.

12. Yoshida H, Kim YH, Ozasa H, Nagai H, Sakamori Y, Nakaoku T, et al. Albumin-bound paclitaxel for the treatment of refractory or relapsed small-cell lung cancer. Mol Clin Oncol. 2016;5:213-215.

13. Si X, Zhang L, Wang H, Zhang X, Wang M, Han B, et al. Management of anlotinib-related adverse events in patients with advanced non-small cell lung cancer: Experiences in ALTER-0303. Thorac Cancer. 2019;10:551-556.

14. Chi Y, Fang Z, Hong X, Yao Y, Sun P, Wang G, et al. Safety and Efficacy of Anlotinib, a Multikinase Angiogenesis Inhibitor, in Patients with Refractory Metastatic Soft-Tissue Sarcoma. Clin Cancer Res. 2018;24:5233-5238.

15. Sun Y, Niu W, Du F, Du C, Li S, Wang J, et al. Safety,pharmacokinetics, and antitumor properties of anlotinib, an oral multi-target tyrosine kinase inhibitor, in patients with advanced refractory solid tumors. J Hematol Oncol.2016;9:105.

16. Li X, Ai B, Zhang P, Li L, Wu X. [Clinical Research on Albumin-bound Paclitaxel-based Therapy in Advanced Lung Cancer]. Zhongguo Fei Ai Za Zhi. 2017;20:479-484. 


\section{Figure legends:}

Table 1 Clinical characteristics of ES-SCLC patients

Table 2 Efficacy profile of the two groups.

Table 3 Safety profile of the two groups.

Fig. 1 PFS and OS curves of Anlotinib and Nab-P groups. (A) Kaplan-Meier survival curves showing the PFS of patients in the Anlotinib group $(n=35)$

and Nab-P group $(\mathrm{n}=36)$ (median PFS, 5.0 vs. 5.0 months; HR [95\% CI]: 0.377 [4.26 -5.74], $\mathrm{p}=0.664$ ); (B) Kaplan-Meier survival curves showing the OS of patients in the Anlotinib group $(\mathrm{n}=35)$ and Nab-P group $(\mathrm{n}=36)$ (median OS, 9.4 vs. 9.2 months; HR [95\% CI]: 0.996 [7.45-11.35], $\mathrm{p}=0.627$ )

Fig. 2 Subgroup analysis of PFS and OS in enrolled patients. Dots and horizontal lines represent the mean value and confidence intervals, respectively. (A) Subgroup analysis of PFS of patients in the Anlotinib group $(n=35)$ and Nab-P group $(n=36)$; (B) Subgroup analysis of OS of patients in the Anlotinib group $(\mathrm{n}=35)$ and Nab-P group $(\mathrm{n}=36)$. 

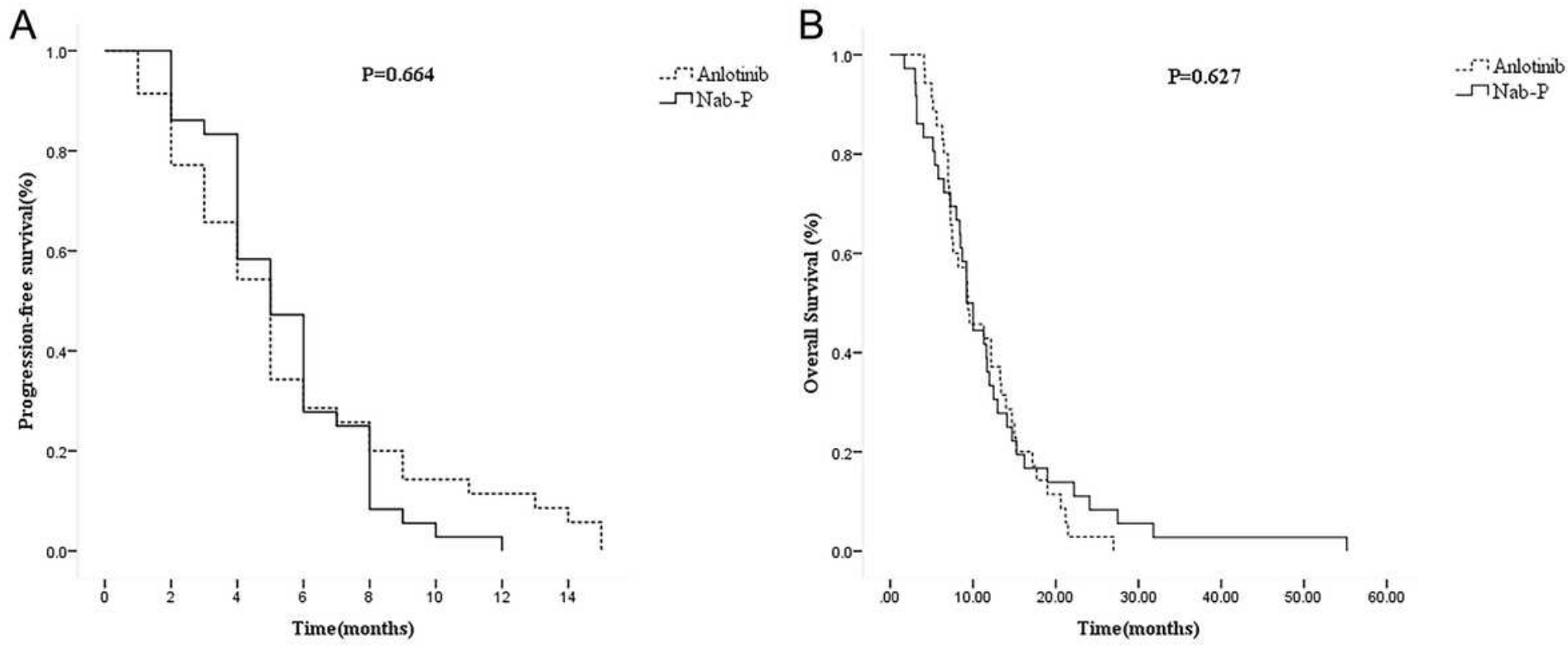

Figure 1

PFS and OS curves of Anlotinib and Nab-P groups. (A) Kaplan-Meier survival curves showing the PFS of patients in the Anlotinib group $(n=35)$ and Nab-P group $(n=36)$ (median PFS, 5.0 vs. 5.0 months; HR [95\% Cl]: 0.377 [4.26 - 5.74], $p=0.664$ ); (B) Kaplan-Meier survival curves showing the OS of patients in the Anlotinib group $(n=35)$ and Nab-P group $(n=36)$ (median OS, 9.4 vs. 9.2 months; HR [95\% Cl]: 0.996 [7.45-11.35], $p=0.627$ )

A

Study
ID

B

Study
ID

\section{Figure 2}


Subgroup analysis of PFS and OS in enrolled patients. Dots and horizontal lines represent the mean value and confidence intervals, respectively. (A) Subgroup analysis of PFS of patients in the Anlotinib group $(n=35)$ and Nab-P group $(n=36)$ (B) Subgroup analysis of OS of patients in the Anlotinib group ( $n$ $=35)$ and Nab-P group $(n=36)$. 